\title{
ZigBee-based Parameter Monitoring and Controlling Scheme for Multiple DC Motors
}

\author{
Naqeeb Ullah ${ }^{1}$, Muhammad Shakeel Akram ${ }^{1}$, Hameed Ullah Buzdar ${ }^{1}$, \\ Atiq Ur Rehman ${ }^{1}$, Muhammad Abbas Khan ${ }^{1, *}$ and Saifullah Khan ${ }^{2}$ \\ ${ }^{1}$ Faculty of Information and Communication Technology, Balochistan University of \\ Information Technology, Engineering and Management Sciences (BUITEMS), Quetta, \\ Pakistan \\ 2School of Electrical Engineering, Universiti Teknologi Malaysia, Skudai, 81310, Johor \\ Bahru, Malaysia
}

Article Type: Article

Article Citation: Naqeeb Ullah, Muhammad Shakeel Akram, Hameed Ullah Buzdar, Atiq Ur Rehman, Muhammad Abbas Khan, Saifullah Khan. ZigBee-based parameter monitoring and controlling scheme for multiple DC motors. Indian Journal of Science and Technology. 2020; 13(06),725-734.D0l:10.17485/ijst/2020/ v013i06/149098

Received date: November 22, 2019

Accepted date: January 24, 2020

*Author for correspondence: Muhammad Abbas Khan Muhammad.Abbas@buitms.edu. pk 9 Faculty of Information and Communication Technology, Balochistan University of Information Technology, Engineering and Management Sciences (BUITEMS), Quetta, Pakistan

\begin{abstract}
Objective/aim: This study suggests a safe and economical wireless control and monitoring system for multiple DC motors based on ZigBee wireless communication.
\end{abstract}

Methods: A set of sensors is used to control and monitor the information regarding the multiple DC motors parameters such as voltage, speed, temperature, and over-current and then transmit this information through the wireless ZigBee Protocol. A microcontroller is used for information collection and storage. According to monitored information, the DC motor will be stopped or started by the control signal via the computer interface produced with ZigBee when a certain condition is accomplished. Findings: The developed system can perform various operations like the automatic on/off of multiple DC motors, forward \& reverse, speed control, checking overload condition, current measuring, and voltage measuring. The parameters: voltage, current, temperature, revolution per minute (RPM) can be detected in real-time by sensors and transferred to the host computer, displayed and store in an excel file. A ZigBee device is used to receive and transmit data efficiently from microcontroller to the PC and vice versa. In the past, similar systems were used for a single DC motor which was expensive such as PLC and Wi-Fi. In this work, a cost-effective ZigBee-based system is used for multiple DC motors. Applications/improvements: The proposed system can be further improved and modified for the real-time measuring system for AC machines. Moreover, the proposed wireless monitoring system can be enhanced with artificial intelligence.

Keyword: DC Motor, Wireless Control and ZigBee Module, Microcontroller, Monitoring System. 


\section{Introduction}

Direct current (DC) motors play a vital role in industrial applications because of their plain, secure configuration, and vast applications in industry. Hence, it becomes necessary to automate and guard them against flaws to assure unbroken operation. DC motors control methods are very sensitive to motor parameters. Numerous controlling methods have been demonstrated to acquire an improved controlling system for them. The monitoring techniques for DC motors can be categorized as conventional and digital techniques. If conventional and digital monitoring is compared, conventional methods significantly decrease the competence and sensitivity of the system because the apparatus increases the time for detecting defects such as electrical and mechanical parameters. Currently, automation has become a crucial need for the industries to have an accurate and precise operation. This study presents a wireless monitoring system using ZigBee wireless communication, granting protected and cost-effective data in industrial areas, wherever wired connectivity is more difficult due to physical constraints [1-2]. The parameters of the DC motors are controlled by a transducer or detector unit, and the data are transmitted through the ZigBee Protocol.

Wireless sensor networks have been familiar in many fields, including automotive information transfer and process control applications [3]. Multiple sensors are used to evaluate and protect the parameters of the DC motor from faults to ensure uninterrupted operation and work. Different techniques have been proved to monitor and control the basic parameter of DC machines. The methods for tracking DC machines can be categorized as conventional and digital techniques. When conventional and digital surveillance is contrasted, conventional techniques considerably reduce the system's complexity and sensitivity due to the amount of electrical and mechanical devices involved in the detection of defects.

In wireless communication using ZigBee, $\mathrm{Wi}-\mathrm{Fi}$, and Bluetooth is the short-range wireless communication system [4]. ZigBee-based Parameter Monitoring and Controlling System for Multiple DC Motors is established on wireless communication through ZigBee a fair way to monitor the key parameters such as voltage, current, revolution per minute (RPM), torque, temperature and control the parameters like ON/OFF, speed, over-voltage, over-current, over-temperature, overload, no-load, Forward and Reverse directions of DC motor. The ZigBee wireless communication is an economical, safe, low-power, high hardware integration and sufficient-data rate wireless communication technology introduced via the ZigBee Alliance [5-6]. For Human it is a difficult task to monitor the parameters continuously, and also to remove the faults from a parameter, which may damage the motor in a wired system. The proposed work offers an advanced solution to monitor and control the essential parameters of various DC motors in real-time.

A microcontroller actively monitors and store information and then produces a control signal to stop and start the DC motors wirelessly via a computer interface built with ZigBee. Moreover, it is also responsible for conducting operations such as operating the device via $\mathrm{RF}$, halting it, calculating, and tracking and controlling most of the motor's parameters such as currents, voltages, the temperature of the wire, rate. It is possible to transfer all these values to the host computer, displayed on a computer screen. This ZigBee module 
is extensively used in numerous applications for its enhanced consistency, power-efficient profile, exceptional capability, high elasticity, and low cost [7]. The proposed system can be applied for industrial applications and beneficial for professional, technical and advanced training in colleges and research institutes.

The proposed method consists of Hardware Design, software programming, and wireless communication system.

\section{ZigBee-based Wireless Communication}

ZigBee-based wireless network not only reduces the cost of labor for monitoring but also provides flexibility in terms of extension of the network. This study proposes a method of wirelessly monitoring and controlling system for multiple DC Motors using ZigBee. It is a new way of monitoring and controlling wirelessly the industrial parameters like the speed of a motor, turn off, turn on, the temperature of winding, forward and reverse moving of motor. Wireless communication deals with the transmission of the data to the ground unit. There are many wireless communication protocols such as Wi-Fi, Bluetooth, and ZigBee. A Protocol is necessary for communicating a device to other devices in the same language for communication across the media which is called a protocol. The Protocol determines how data can be transmitted. The ZigBee protocol is standardized under IEEE 802.50.4 standard. It affords a low data rate and as well as low power utilization [8]. In [9] fact, a ZigBee is the development of the physical and MAC layers. The term ZigBee comes into play from the honeybee, for communication they make use of a zigzag style dance. ZigBee outline envelops little energy, house, sanatorium along with user electronic supervising, measuring, monitoring, and controlling the essential parameters of the industrial machines. The wireless sensor network frequently utilizes the ZigBee protocol because of its intelligent network by using mesh topology in which the sensors are capable to communicate with each other regardless of direction- finding device. The ZigBee that pursues the IEEE 802.15.4 standard classified as PWAN that stands for personal wireless area network operating in two ISM (Industrial, scientific, medical) band at $915 \mathrm{MHz}$ and $2.4 \mathrm{GHz}$. The ISM band is unlicensed. ZigBee data rate that is achieved is round about 250 $\mathrm{Kb} / \mathrm{s}$. A comprehensive summary of the programmable logic controller (PLC), Bluetooth, $\mathrm{Wi}-\mathrm{Fi}$, and ZigBee has been given in Table 1.

It can be concluded from Table 1 that ZigBee is better and reliable for industrial applications. ZigBee has less power consumption with wireless technology, easy installation and error finding is too much easy among the number of nodes to be connected with ZigBee. As in industry PLC is used a lot, but has disadvantages like power consumption, difficulty in trace finding the error, difficulty in installation.

\section{Monitoring and Controlling Scheme}

The section highlights the monitoring and control scheme for multiple DC motors. The schematic diagram of the planned system is illustrated in Figure 1. The entire hardware 
TABLE 1. Comparison of PLC Bluetooth, Wi-Fi, and ZigBee

\begin{tabular}{lllll}
\hline Key parameters & PLC & Bluetooth & Wi-Fi & ZigBee \\
\hline Connections & Wired & Wireless & Wireless & Wireless \\
Power consumption & High & Low & Medium & Very low \\
Range & - & $10 \mathrm{~m}$ & $100 \mathrm{~m}$ & $10-100 \mathrm{~m}$ \\
Installation & Difficult & Easy & Easy & Easy \\
Error finding capability & Difficult & Easy & Easy & Easy \\
Number of nodes & - & 8 & 2007 & $>65,000$ \\
Data rates & - & $1 \mathrm{Mb} / \mathrm{s}$ & $54 \mathrm{Mb} / \mathrm{s}$ & $250 \mathrm{~Kb} / \mathrm{s}$ \\
\hline
\end{tabular}

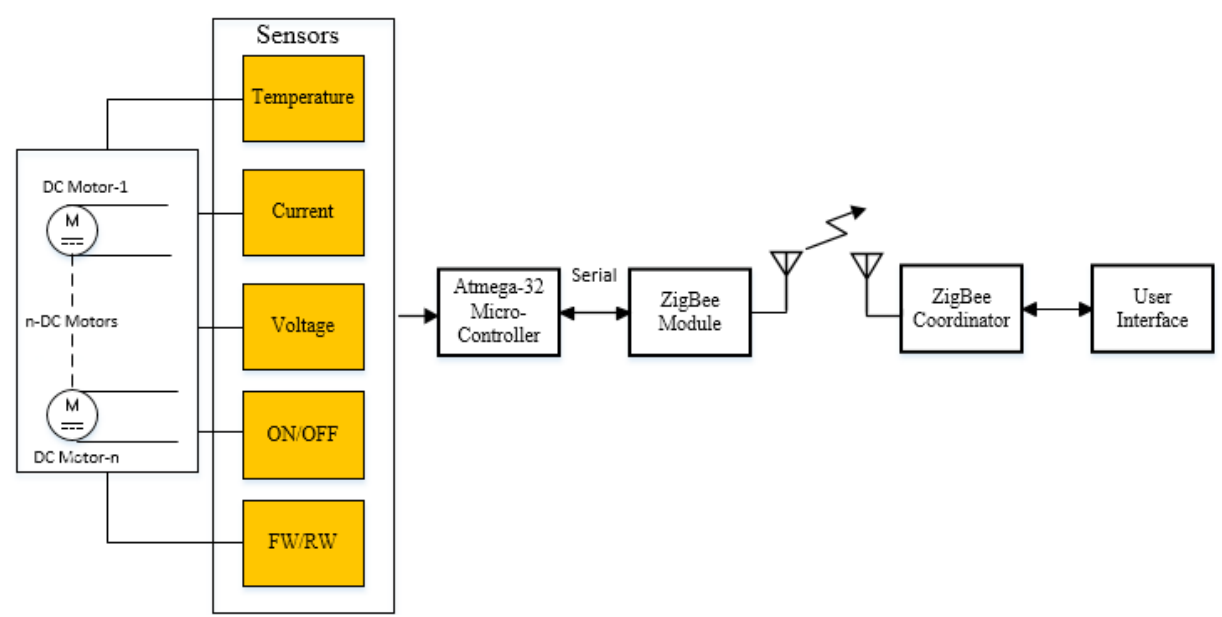

FIGURE 1. Schematic diagram of ZigBee-based parameter monitoring and controlling system.

system has been divided into two parts - the machine part and the control room part. A sensor network is employed in the machine part to monitor parameters such as voltage, current, stator winding temperature, and DC motor speed. Various sensors have been used to monitor the data and instantaneously fed to the microcontroller. These data are well transmitted to the control room side via the ZigBee protocol. The microcontroller on the machine part side is programmed so that if the monitoring parameter values of the DC device exceed the necessary or protective threshold, a control signal will be made by the microcontroller [10].

All the parameters can be observed in real-time through a visual Basics Graphical User interface (GUI) on a personal computer. The GUI is developed by the client depending on the implementation. All processors and sensors are connected through ZigBee to an external PC. The Personal Computer will also continuously display and compare each of the Data from the distant preparation facility.

\section{Hardware Description}

The proposed method consists of several hardware parts and can be divided into two subsections: Transmitter module, and Receiver Module. The transmitter module consists 
of several sensors and circuits to measure the proposed parameters of the DC machine. In this research work, we concentrated on the fundamental parameters of DC machines including temperature, current, voltage, automatic on/off and speed and motor direction.

\subsection{Temperature Sensor}

In this research work, the LM 35 semiconductor temperature sensor is used to monitor the temperature of DC motors. The output voltage is linearly proportional to the Celsius (Centigrade) temperature. The LM35 produces higher output voltage rather than a thermistor and not require amplifying the output voltage.

\subsection{Current Sensor}

This sensor is used to measure the current of the DC motor. If the current measured is increased or decreased to the pre-set value, then a signal will be received on the PC and further proper action is taken on it.

\subsection{Voltage Sensor}

The voltage sensor continuously monitors the voltage of the DC motor. If the voltage is over concerning a predetermined value, then the microcontroller system gives a control signal to stop the motor.

\subsection{Inductive Proximity Sensor}

The speed of the revolution of the DC motor can be measured using the inductive proximity sensor. An inductive proximity sensor is a metal sensor that senses the presence and absence of the metal on which generates a pulse. By that pulse, we calculate the RPM of the motor.

\subsection{Atmel Microcontroller (ATMEGA 32)}

A microcontroller from ATMEL is power-driven by the AVR core. ATMEGA 32 is one new mega ranges of Atmel AVR microcontroller and imperative offering large space program memory. The ATMEGA 32 consists of a large $32 \mathrm{~KB}$ of program flash memory and crystal oscillator which consists of $16 \mathrm{MHz}$, which resets switch and connector for LCD and ZigBee Module Interfacing. In this research work, the ATMEGA 32 is used because of a variety of features such as some additional peripherals, 3 flexible timer/counters with compare modes. Moreover, it can be programmed by C-language, assembly language, or high level/low-level language [11]. The input level can be modified to generate an interrupt Added in EEPROM to store data over power-off time, needed program space for additional parameter program can be added and most important at mega have faster execution speed other than a microcontroller. 


\subsection{ZigBee $T X$ and $R X$}

The ZigBee RF modules are designed to operate within the ZigBee protocol and support the unique needs of low cost, low power and a large number of nodes connecting through ZigBee. The module can operate on the ISM $2.4 \mathrm{GHz}$ frequency band within a range of 100-200 m. The receiver side module is connected to the computer system to the serial cable RS232. Thus, the monitoring data received by the ZigBee module are directly transferred to the PC and result display if the form of GUI. The ZigBee module has been shown in Figure 2.

\section{Proteus 8 Professional Software}

In the research work, a Proteus 8 professional is used to model the proposed design. The direct implementation of codes on a microcontroller may damage or burn it. So it is useful to firstly design the circuit on Proteus software before implementing it on the microcontroller. It has the versatility of dealing string and many other functions that make it the great programming language over other programming software.

\subsection{Circuit Designing on Proteus ISIS}

The circuits are designed on Proteus using this ISIS schematic package. It provides a powerful design environment and can define most aspects of the drawing appearance. The schematic diagram of the proposed system is illustrated in Figure 3.

ATMEGA 32 microcontroller is used for controlling the parameters of multiple DC motors. Moreover, Micro C pro is used for the coding of the microcontroller as it is easy and requires less time to code and can easily understand. It also provides the conversion

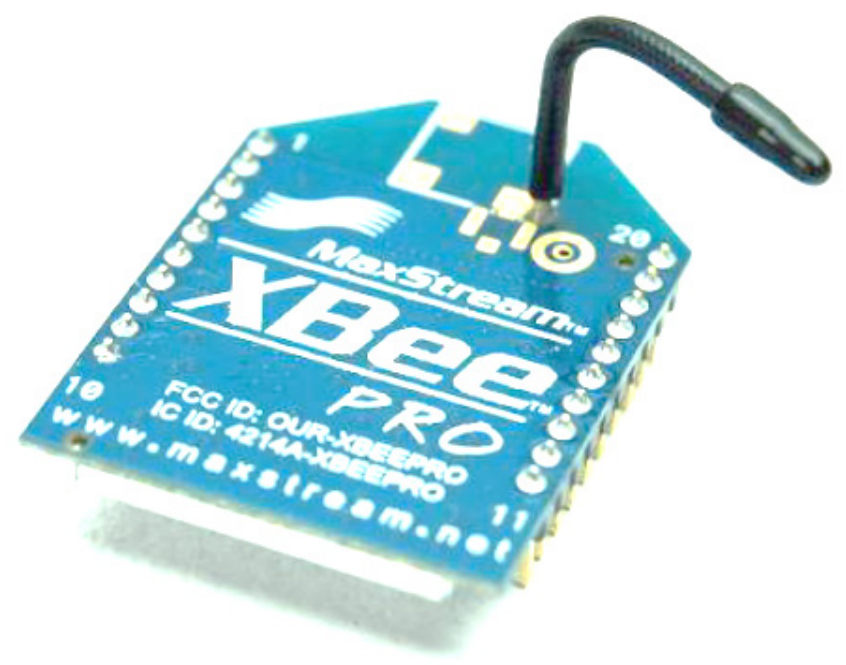

FIGURE 2. ZigBee pro module. 


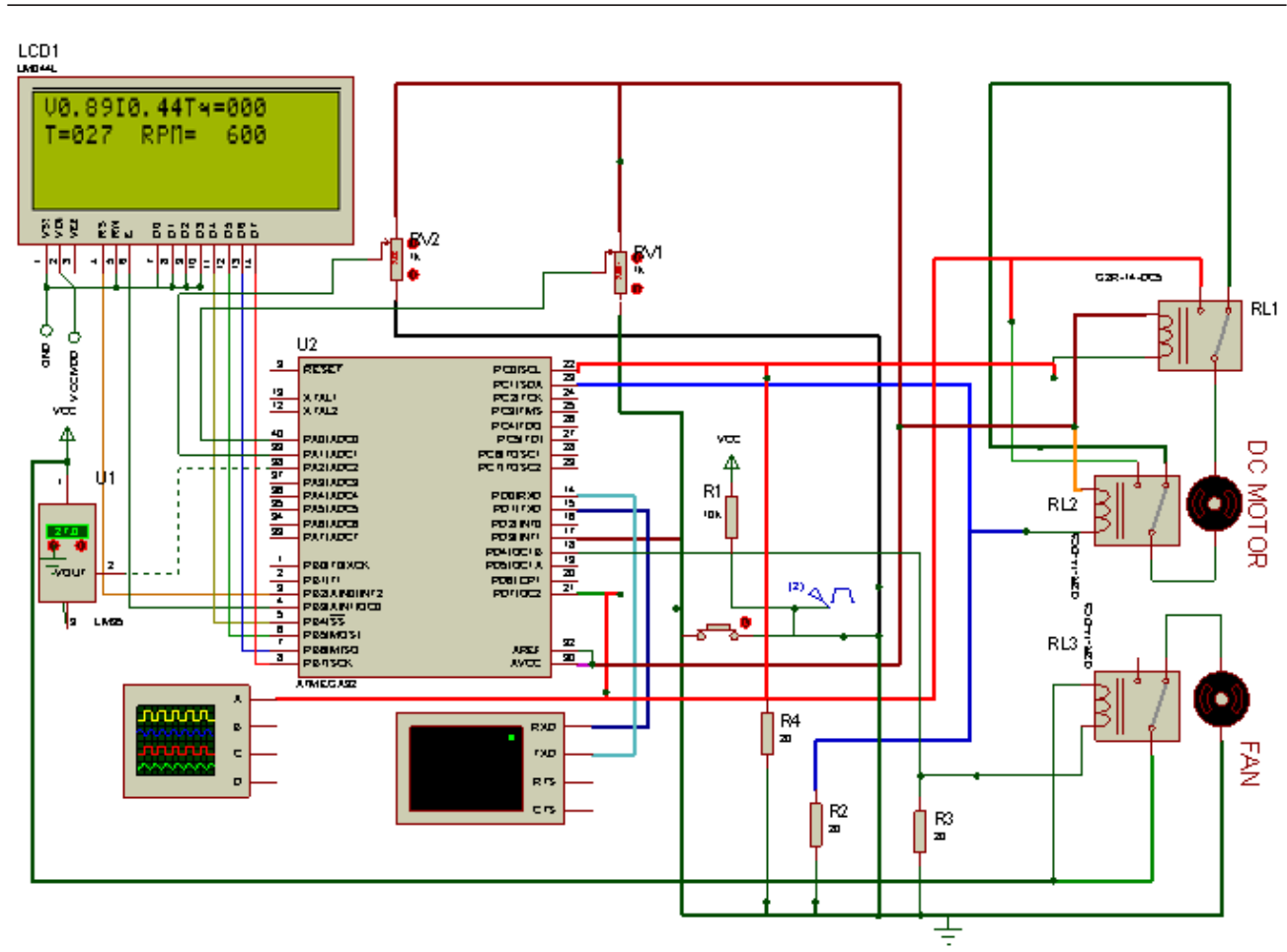

FIGURE 3. Schematic diagram of the proposed system in Proteus ISIS.

from $\mathrm{C}$ language programming to Assembly language programming with less difficulty. A new project is created, coded, compiled, and tested the results.

\subsection{AVR FLASH}

The program is burned into the microcontroller using a burner. It is possible to program any AVR microcontroller, by using AVR flash burner software for programming. The AVRFLASH programmer is connected to the microcontroller via six lines, i.e. (VCC, GND, RESET, SCK, MOSI, MISO) targeted ATMEGA 32 which is placed in the breadboard or a burner. 3.4.1. Flash Burning Process includes the start of AVR Loader and selects the microcontroller and afterward point out the hex file and click Write Button (in the "Flash" option). When the microcontroller is programmed and the file is saved, those files end in .hex (short for Intel Hex Format) and .hex file is generated this file is just required to send to the microcontroller chip.

\section{Result \& Discussion}

Figure 4 illustrates the flow chart of the proposed system. Multiple sensors are used to sense DC Motors parameters including voltage, current, temperature, speed. Initially, when the system starts it goes on the standby mode, initializes the whole system. 


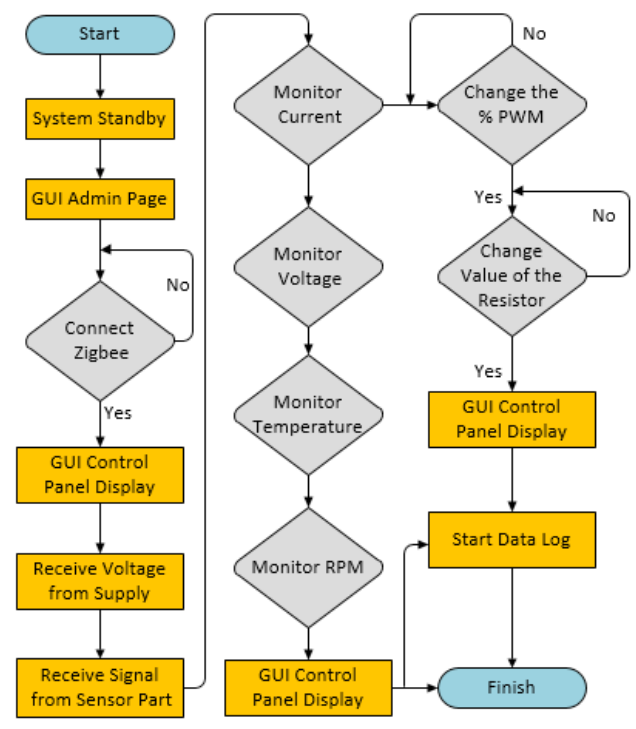

FIGURE 4. Flow chart of the working principle of the proposed system.

After that, it displays the welcome messages "WELL COME MOTOR" on LCD. After a small delay, it displays a message on the LCD "RHNSSI GROUP PRODUCTION" and then it shows another message on the laptop screen "WELL COME 1" and the measured parameters and will send it to the user end computer as illustrated in Figure 5.

The results are sent to the host computer via ZigBee-based wireless communication. Moreover, the received data are stored into a database and can be monitored in real-time on $\mathrm{X}$-CTU (GUI) as well as on LCD. If any parameter exceeds the threshold or set commands, a control signal will be generated and the microcontroller will perform necessary action. The hardware implementation is illustrated in Figure 6 where various sensors, monitoring, and control circuits are integrated.

The proposed system is flexible and can easily extend by adding more sensors and control circuits to monitor more parameters.

\section{Conclusion}

In this study, we proposed a system for remote monitoring and controlling of DC motors parameter using ZigBee technology. The designed system can be used for industrial applications and also for educational purposes in universities, colleges, and schools. The developed system can perform different operations on DC motors like motor start \& stop, forward \& reverse, speed control, checking overload condition, current measuring, voltage measuring, and speed. The parameters: voltage, current, temperature, RPM can be detected by sensors and transferred to the host computer, displayed and store in an excel file. The ZigBee device is used to receive and transmit data from the microcontroller to the PC and from PC to the Microcontroller. A ZigBee control system is designed according 


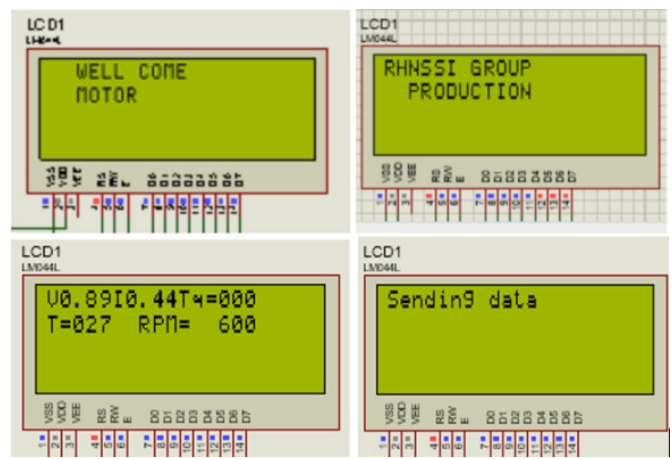

FIGURE 5. Welcome messages on LCD.

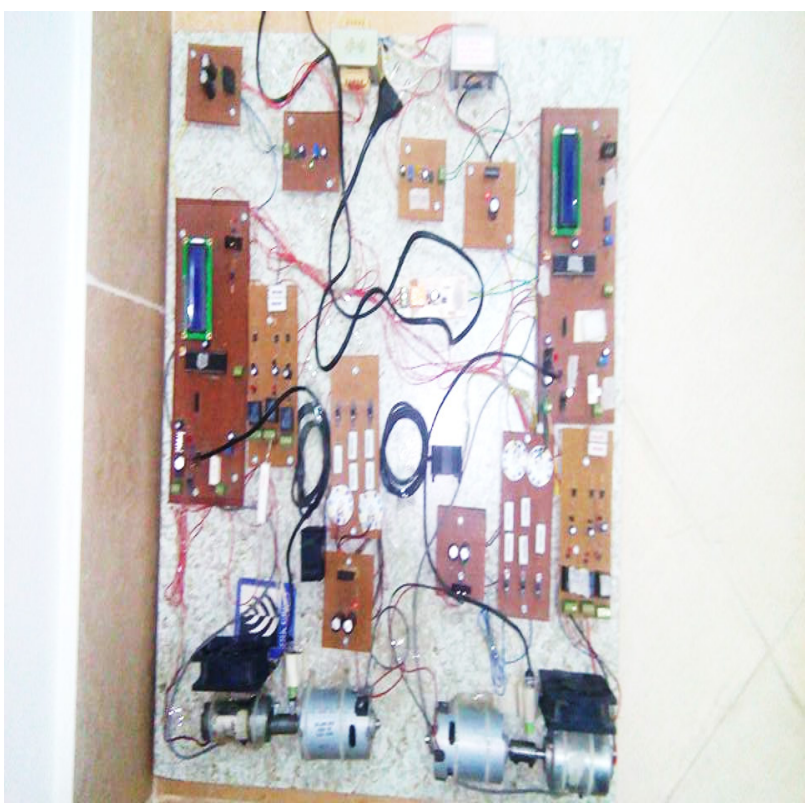

FIGURE 6. The experimental set-up of monitoring and controlling system for multiple DC motors using ZigBee technology.

to a modular structure during the programming steps. This system is also helpful for the researcher, as they are performing experimental researches in laboratories.

\section{References}

1. Mercier D, Sibuet H, Dieppedale C, Baret C, Bonnard C, Billard C. Analysis of a thin film dielectric characterization method based on the impedance difference of two MIM capacitors. International Journal of Current Engineering and Technology. 2017, 85-8. http://inpressco.com/ wp-content/uploads/2013/11/Paper131667-1671.pdf

2. Kothari DA், Manish Thakker $\dot{A} \dot{B}$, Shah $\dot{A} V$, Gohel T. A real-time wireless multi-parameterer monitoring system with ZigBee and LabVIEW. International Journal of Current Engineering 
and Technology. 2013, 3(5), 1667-1671. https://www.academia.edu/6064023/A_Real_Time_ Wireless_Multi_Parameter_monitoring_System_with_ZigBee_and_LabVIEW

3. Patil RR, Date TN, Kushare BE. ZigBee based parameters monitoring system for induction motor. IEEE Students' Conference on Electrical, Electronics and Computer Science. 2014, 23362339. https://doi.org/10.1109/SCEECS.2014.6804469

4. Danbatta SJ. Comparison of Zigbee, Z-Wave, Wi-Fi, and Bluetooth wireless technologies used in home automation. In: 7th international symposium on digital forensics and security. 2019; 1-5. https://doi.org/10.1109/ISDFS.2019.8757472

5. Saleem Y, Member S, Crespi N, Member S. Internet of things-aided smart grid: technologies, architectures, applications, prototypes, and future research directions. IEEE Access. 2019, 7, 62962-63003. https://doi.org/10.1109/ACCESS.2019.2913984

6. Zhu Q. Data acquisition technology of air pollution sources in ecological monitoring database. Ekoloji. 2019, 28(107), 3035-3044. http://ekolojidergisi.com/download/data-acquisitiontechnology-of-air-pollution-sources-in-ecological-monitoring-database-5940.pdf

7. Myint CZ, Gopal L, Aung YL. WSN-based reconfigurable water quality monitoring system in an IoT environment. In: 14th international conference on electrical engineering/electronics, computer, telecommunications and information technology (ECTI-CON). 2017; 741-744. https://doi.org/10.1109/ECTICon.2017.8096345

8. Gočal P, Macko D. Eemip: Energy-efficient communication using timing channels and prioritization in Zigbee. Sensors. 2019, 19(10), 2246. https://www.ncbi.nlm.nih.gov/pmc/ articles/PMC6567886/

9. Ejaz W, Anpalagan A. Communication technologies and protocols for internet of things. Internet of Things for Smart Cities. 2019, 17-30. https://link.springer.com/chapter/10.1007/ 978-3-319-95037-2_2

10. Malhi K, Mukhopadhyay SC, Schnepper J, Haefke M, Ewald H. A Zigbee-based wearable physiological parameters monitoring system. IEEE Sensors Journal. 2012, 12(3), 423-430. https://doi.org/10.1109/JSEN.2010.2091719

11. Dalef HH, Aziz FA, Hasan WZW, Ariffin MKAM. Development of wireless controlling and monitoring system for the robotic hand using Zigbee protocol. Journal of Computational and Theoretical Nanoscience. 2018, 15(2), 656-662. https://doi.org/10.1166/jctn.2018.7140 